\title{
Cost-Effectiveness of Antiretroviral Therapy for Multidrug-Resistant HIV: Past, Present, and Future
}

\author{
Marianne Harris, ${ }^{1,2}$ Bohdan Nosyk, ${ }^{3}$ Richard Harrigan,, ${ }^{3,4}$ Viviane Dias Lima, ${ }^{3,4}$ \\ Calvin Cohen, ${ }^{5}$ and Julio Montaner ${ }^{3,4}$ \\ ${ }^{1}$ AIDS Research Program, Providence Health Care Institute, $B 5181081$ Burrard Street, Vancouver, BC, Canada V6Z 1 Y6 \\ ${ }^{2}$ Department of Family Practice, Faculty of Medicine, University of British Columbia, Vancouver, BC, Canada V6T $1 Z 3$ \\ ${ }^{3}$ British Columbia Centre for Excellence in HIV/AIDS, Vancouver, BC, Canada V6Z $1 Y 6$ \\ ${ }^{4}$ Division of AIDS, Faculty of Medicine, University of British Columbia, Vancouver, BC, Canada V6T $1 Z 3$ \\ ${ }^{5}$ Community Research Initiative of New England, Boston, MA 02111, USA
}

Correspondence should be addressed to Marianne Harris, mharris@cfenet.ubc.ca

Received 17 January 2012; Revised 1 October 2012; Accepted 2 October 2012

Academic Editor: Giuseppe Ippolito

Copyright (C) 2012 Marianne Harris et al. This is an open access article distributed under the Creative Commons Attribution License, which permits unrestricted use, distribution, and reproduction in any medium, provided the original work is properly cited.

\begin{abstract}
In the early years of the highly active antiretroviral therapy (HAART) era, HIV with resistance to two or more agents in different antiretroviral classes posed a significant clinical challenge. Multidrug-resistant (MDR) HIV was an important cause of treatment failure, morbidity, and mortality. Treatment options at the time were limited; multiple drug regimens with or without enfuvirtide were used with some success but proved to be difficult to sustain for reasons of tolerability, toxicity, and cost. Starting in 2006, data began to emerge supporting the use of new drugs from the original antiretroviral classes (tipranavir, darunavir, and etravirine) and drugs from new classes (raltegravir and maraviroc) for the treatment of MDR HIV. Their availability has enabled patients with MDR HIV to achieve full and durable viral suppression with more compact and cost-effective regimens including at least two and often three fully active agents. The emergence of drug-resistant HIV is expected to continue to become less frequent in the future, driven by improvements in the convenience, tolerability, efficacy, and durability of first-line HAART regimens. To continue this trend, the optimal rollout of HAART in both rich and resource-limited settings will require careful planning and strategic use of antiretroviral drugs and monitoring technologies.
\end{abstract}

\section{Introduction}

In the early years of the highly active antiretroviral therapy (HAART) era starting in 1996, HIV with resistance to two or more agents in different antiretroviral classes posed a significant clinical challenge. Multidrug-resistant (MDR) HIV was an important cause of treatment failure and consequent morbidity and mortality [1]. In 1998, a large drug resistance survey among viremic HIV patients in the United States showed that $13 \%$ harbored three-class-resistant virus and $48 \%$ had two-class resistance [2]. With improvements in understanding of viral dynamics and the efficacy of firstline regimens, MDR HIV has become less common but has not disappeared entirely, as demonstrated in a Canadian cohort of HAART-treated individuals followed until 2007
[3]. While three-class antiretroviral drug resistance is now very unusual (2\%), two-class resistance was observed in $17 \%$ of the cohort. Fortunately, treatment options for patients with MDR HIV have improved substantially in terms of effectiveness, toxicity, and tolerability, while remaining costeffective in most cases.

\section{Past (1996-2005)}

2.1. The HAART Era. The HAART era began in 1996, with the availability of triple drug regimens and clinical trial data demonstrating their efficacy $[4,5]$. Around the same time, the availability of viral load testing improved both the understanding of viral dynamics in response 
to treatment and the ability to closely monitor treatment efficacy. The consequences of exposure to sequential drug regimens in the absence of full viral suppression were not fully appreciated until the advent of widespread HIV drug resistance testing around 2000. Most of the HIV-infected patients who had previously received less effective single and dual drug regimens had already developed drug-resistant HIV by this time. Furthermore, some early triple therapy regimens were less than optimally effective, due to relatively low potency of individual drugs and adherence challenges related to complex dosing with numerous pills and poor tolerability. As a result, during this time a significant proportion of treatment-exposed HIV-infected patients harbored MDR strains [2].

2.2. Treatment Strategies for Multidrug-Resistant HIV. In the first decade of HAART, treatment options for MDR HIV were very limited. Before 2003, all available antiretroviral agents belonged to one of the original three drug classes and considerable cross-resistance existed within each class. Given the limited effective drug options available at the time, various strategies were tried. Regimens including two protease inhibitors (PIs), saquinavir and ritonavir both in therapeutic doses, achieved good results in treatmentexperienced patients who had not previously been exposed to PIs [6]; however, results of this dual PI-based regimen were variable in patients who had experienced indinavir or nelfinavir failure previously [7, 8]. Another strategy was to promote reemergence of drug-susceptible virus using structured treatment interruptions and thereby to enhance virologic response to subsequent antiretroviral therapy [9]. This strategy was abandoned when it was proven to be ineffective in promoting sustained virologic suppression or disease control and, more alarmingly, was associated with protracted CD4 declines [10]. A more successful treatment strategy was the use of multiple drug rescue therapy, also called mega-HAART or giga-HAART, whereby patients were treated with as many partially active agents as possible, generally six to eight [11-13]. This strategy proved to be effective for at least some of the patients, but adherence was a significant challenge because of regimen complexity and poor tolerability. Long-term sustainability was limited by toxicity and cost issues.

2.3. Enfuvirtide. In 2003, 24-week results of two large randomized controlled studies ( $\mathrm{T}-20$ versus optimized background regimen only (TORO) 1 and 2) were published that demonstrated the efficacy of enfuvirtide, an HIV fusion inhibitor, for the treatment of patients with drug-resistant virus $[14,15]$ (Table 1$)$. In the combined TORO 1 and 2 studies, the 48 -week rates of virologic suppression to $<50$ copies/mL were $18.3 \%$ for enfuvirtide plus an optimized background regimen versus $7.8 \%$ for the optimized background regimen alone [16]. The drug received regulatory approval in Canada, the United States (US), and Europe in the same year (Table 2). Enfuvirtide represented an important breakthrough in that it was the first approved antiretroviral agent belonging to a new drug class, and hence cross-resistance with previous agents was not a problem. By the middle of the decade, enfuvirtide was considered a cornerstone of treatment for patients harboring MDR virus [17].

However, the complexity of synthesis and limited supply led to pricing of enfuvirtide in the US and Europe at $\$ 18,500$ per person per year (calculated in 2001 US dollars, from $\$ 20,000$ dollars in 2003 using the medical care component of the consumer price index), which was nearly twice as costly as any of the other approved single agents for treatment of HIV in use at the time [18-22]. In addition, enfuvirtide had to be used in combination with multiple other active antiretroviral agents $[16,23]$. As a result, the annual cost of a combination antiretroviral regimen containing enfuvirtide was typically between $\$ 35,000$ and $\$ 43,000$ per person per year $[18,24]$.

Extrapolating from the 24- and 48-week TORO results, the cost-effectiveness of enfuvirtide in combination antiretroviral regimens was evaluated by Sax et al. in 2005 [25] and by Hornberger et al. in 2006 [26]. Using different methods of analysis, these two papers estimated the incremental cost-effectiveness ratio of enfuvirtide plus an optimized background regimen compared with an optimized background regimen alone to be $\$ 69,500$ and $\$ 24,604$, respectively, per quality-adjusted life-year (QALY) gained. (To calculate the incremental cost-effectiveness of an intervention, analyses must consider the added efficacy (generally measured in quality-adjusted life years (QALY)) and the additional costs of the intervention. The costeffectiveness ratio is then calculated with incremental costs in the numerator and incremental benefits in the denominator (\$/QALY). An intervention may be considered cost-effective if the additional benefit provided by the treatment is considered "worth" the additional cost. The World Health Organization (WHO) Commission on Macroeconomics and Health suggested that interventions may be considered very cost-effective when the costeffectiveness ratio (\$/QALY) is less than 1 times the percapita gross domestic product (GDP) for an individual country and cost-effective when the ratio is less than 3 times the per-capita GDP [27]. As a point of reference, the estimated GDP per capita in Canada in 2010 is $\operatorname{CDN} \$ 39,057$ [28].)

The results of these studies suggested that enfuvirtidebased regimens could represent a cost-effective option for treating individuals with MDR HIV and advanced disease at the time. The projected survival benefit of enfuvirtide plus an optimized background regimen becomes more apparent with longer-term followup [26]; however, longterm sustainability of enfuvirtide therapy was hampered by the need for twice daily subcutaneous injections and bothersome injection site reactions $[29,30]$. Because of these issues and the availability of newer, more convenient oral agents, enfuvirtide is no longer widely used; however, there is no doubt that this agent saved the lives of many MDR-HIVinfected patients who would otherwise not have survived until other more sustainable options became available. 
TABle 1: Publications of pivotal studies of drugs for multidrug-resistant HIV.

\begin{tabular}{|c|c|c|c|}
\hline Drug & $\begin{array}{l}\text { Abbreviated study title } \\
\text { and duration }\end{array}$ & Study treatment $(N)$ and comparator $(N)$ arms & $\begin{array}{l}\text { Journal and date of } \\
\text { publication }\end{array}$ \\
\hline \multirow{5}{*}{ Enfuvirtide (ENF) } & TORO 1 (24 wks) & $\mathrm{ENF}+3-5$ drug OBT (328) versus 3-5 drug OBT (167) & NEJM, May 2003 [14] \\
\hline & TORO 2 (24 wks) & ENF + 3-5 drug OBT (335) versus 3-5 drug OBT (169) & NEJM, May 2003 [15] \\
\hline & TORO 1 and 2 & & \\
\hline & 48 wk efficacy & $\mathrm{ENF}+\mathrm{OBT}(661)$ versus OBT (334) & $\begin{array}{l}\text { JAIDS, December } 2005 \\
{[16]}\end{array}$ \\
\hline & 48 wk safety & $\mathrm{ENF}+\mathrm{OBT}(663)$ versus OBT $(334)$ & $\begin{array}{l}\text { JAIDS, December } 2005 \\
{[29]}\end{array}$ \\
\hline \multirow{2}{*}{ Tipranavir (TPV) } & RESIST (48 wks) & TPV/r + OBT (746) versus CPI/r + OBT (737) & Lancet, August 2006 [31] \\
\hline & POWER 1 (24 wks) & $\mathrm{DRV} / \mathrm{r}$ in 1 of 4 doses + OBT (255) versus $\mathrm{CPI} / \mathrm{r}+\mathrm{OBT}(63)$ & AIDS, February 2007 [37] \\
\hline \multirow{3}{*}{ Darunavir (DRV) } & POWER 2 (24 wks) & $\mathrm{DRV} / \mathrm{r}$ in 1 of 4 doses $+\mathrm{OBT}$ (225) versus $\mathrm{CPI} / \mathrm{r}+\mathrm{OBT}(53)$ & AIDS, March 2007 [38] \\
\hline & $\begin{array}{l}\text { POWER } 1 \text { and } 2 \\
(48 \text { wks })\end{array}$ & $\begin{array}{l}\mathrm{DRV} / \mathrm{r} 600 / 100 \mathrm{mg} \mathrm{BID}+\mathrm{OBT}(110) \text { versus CPI/r + OBT } \\
(120)\end{array}$ & Lancet, April 2007 [32] \\
\hline & DUET 1 (24 wks) & $\mathrm{ETR}+\mathrm{DRV} / \mathrm{r}+\mathrm{OBT}$ (304) versus DRV/r + OBT (308) & Lancet, July 2007 [33] \\
\hline \multirow[t]{2}{*}{ Etravirine (ETR) } & DUET $2(24 \mathrm{wks})$ & $\mathrm{ETR}+\mathrm{DRV} / \mathrm{r}+\mathrm{OBT}$ (295) versus DRV/r + OBT (296) & Lancet, July 2007 [34] \\
\hline & DUET 1 and 2 (48 wks) & $\mathrm{ETR}+\mathrm{DRV} / \mathrm{r}+\mathrm{OBT}$ (599) versus DRV/r + OBT (604) & AIDS, November 2009 [39] \\
\hline Raltegravir (RAL) & $\begin{array}{l}\text { BENCHMRK } 1 \text { and } 2 \\
\text { (48 wks) }\end{array}$ & $\mathrm{RAL}+\mathrm{OBT}(462)+\mathrm{OBT}(237)$ & NEJM, July 2008 [36] \\
\hline Maraviroc (MVC) & $\begin{array}{l}\text { MOTIVATE } 1 \text { and } 2 \\
(48 \text { wks })\end{array}$ & $\begin{array}{l}\text { MVC QD + OBT (414) versus MVC BID + OBT (426) versus } \\
\text { OBT (209) }\end{array}$ & NEJM, October 2008 [35] \\
\hline
\end{tabular}

OBT: optimized background therapy; CPI: comparator protease inhibitor; r: ritonavir; QD: once daily; BID: twice daily; NEJM: The New England Journal of Medicine; JAIDS: Journal of Acquired Immune Deficiency Syndromes.

TABLE 2: HIV drug approval/authorization dates.

\begin{tabular}{lccc}
\hline Drug & Canada (Health Canada) & US (FDA) & Europe (EMEA) \\
\hline Enfuvirtide & July 14, 2003 & March 13, 2003 & May 5, 2003 \\
Tipranavir & November 21, 2005 & June 22, 2005 & October 25, 2005 \\
Darunavir & July 28, 2006 & June 23, 2006 & February 12, 2007 \\
Maraviroc & September 21, 2007 & August 6, 2007 & September 18, 2007 \\
Raltegravir & November 27, 2007 & October 12, 2007 & December 20, 2007 \\
Etravirine & August 23, 2008 & January 18, 2008 & August 28, 2008 \\
\hline
\end{tabular}

US (FDA): United States Food and Drug Administration; EMEA: European Medicines Agency.

\section{Present (2006-2011)}

Starting in 2006, results of a number of clinical trials involving new antiretroviral agents were published in rapid succession (Table 1). Taken together, these studies represented a significant step forward in the treatment of MDR HIV: tipranavir (RESIST) [31], darunavir (POWER) [32], etravirine (with darunavir in DUET) [33, 34], maraviroc (MOTIVATE) [35], and raltegravir (BENCHMRK) [36]. The regulatory approval of these drugs in Canada, the US, and Europe between 2005 and 2008 enabled prescribers to effectively treat MDR HIV with more compact regimens including at least two and often three fully active agents, with a remarkable increase in the efficacy rates (Table 2). Full and durable viral suppression once again became a realistic goal in the treatment of these patients.

3.1. Tipranavir. Tipranavir was the first of a new generation of ritonavir- ( $\mathrm{r}-$ ) boosted PIs with efficacy against HIV strains that had reduced susceptibility to older PIs, including strains with multiple PI resistance-associated mutations [31]. Using 48-week data from the RESIST studies, Hubben et al. [40] and Simpson et al. [41] demonstrated that regimens including tipranavir/ $\mathrm{r}$ could provide longer-term benefits in terms of reductions in AIDS events and corresponding QALY gains and life years saved, as compared to regimens based on older ritonavir-boosted PIs. These analyses found similar cost-effectiveness ratios for tipranavir/ $\mathrm{r}$ versus comparator $\mathrm{PI} / \mathrm{r}$ of $€ 42,500$ [40] and $\$ 56,517$ [41] per QALY gained. Excluding patients also treated with enfuvirtide reduced the incremental cost-effectiveness ratio to $\$ 46,147$ per QALY [41]. However, the use of tipranavir was limited by important tolerability and toxicity issues, including relatively uncommon but potentially fatal hepatotoxicity and intracranial hemorrhage [42].

3.2. Darunavir. Darunavir, another ritonavir-boosted PI that was also developed to treat PI-resistant HIV, is effective 
and generally safe and well-tolerated. The phase IIb POWER (performance of TMC-114/r when evaluated in treatmentexperienced patients with PI resistance) trials [32, 37, 38 ] and the phase III TITAN (TMC114/r in treatmentexperienced patients naïve to lopinavir) trial [43] demonstrated the efficacy of darunavir/r $600 / 100 \mathrm{mg}$ twice daily among treatment-experienced HIV-infected adults. Subjects in the comparator arms received single $(74 \%)$ or dual (23\%) boosted PIs (mainly lopinavir, saquinavir, and/or amprenavir/fosamprenavir) in POWER and lopinavir/ $\mathrm{r}$ in TITAN. A recent systematic review summarized the results of a number of cost-utility analyses conducted alongside these trials and demonstrated that the use of darunavir/ $\mathrm{r}$ in this setting was cost-effective and, in some cases, cost saving [44].

As a result of complex PI resistance profiles, during this period there was some clinical use of two or more ritonavirboosted PIs in a regimen [45]. A study comparing single to dual unboosted PIs showed modest benefit from the addition of the second PI [46]. The paucity of antiretrovirals from new classes led to clinical use of such dual PI regimens as one way to attempt to reestablish virologic suppression especially in patients with PI resistance. Given the results of the POWER studies, the ability of boosted darunavir to be used in place of a dual boosted PI regimen was explored in two similar randomized controlled trials of immediate substitution of ritonavir-boosted PIs with darunavir/ $\mathrm{r}$ versus deferred substitution after 24 weeks [47, 48]. Together these two pilot-sized studies randomized 48 subjects (24 to each arm) who had undetectable plasma HIV RNA $(<50$ copies $/ \mathrm{mL})$ while receiving regimens including dual or triple boosted PIs. All 45 subjects who completed 24 weeks on study (23 in the immediate switch arms and 22 in the deferred switch arms) had undetectable viral load at week 24. Median CD4 cell count changes from baseline to week 24 were similar in the two arms. At week 48, virologic suppression was maintained in all but two subjects, one in each treatment arm. In this context, darunavir/ $\mathrm{r}$ was shown to be an effective, compact, and relatively safe and tolerable option, as well as being less costly than two or three concomitant ritonavir-boosted PIs.

3.3. Etravirine, Maraviroc, and Raltegravir. The DUET, MOTIVATE, and BENCHMRK trials evaluated the efficacy of etravirine (plus darunavir/r), maraviroc, and raltegravir respectively, versus placebo, each given with an optimized background regimen of nucleoside reverse transcriptase inhibitors, PIs, and/or enfuvirtide [33-36].

Etravirine, the first available next-generation nonnucleoside reverse transcriptase inhibitor, has been successfully used for the treatment of HIV with some degree of resistance to the first-generation nonnucleoside reverse transcriptase inhibitors delavirdine, nevirapine, and efavirenz. Its main use, as supported by data from the DUET study, is in regimens also including a ritonavir-boosted PI, specifically darunavir $[33,34]$. In the combined 48 -week results of the DUET 1 and 2 studies among subjects with nonnucleoside- and PI-resistant HIV, virologic suppression to $<50$ copies $/ \mathrm{mL}$ was observed in $61 \%$ of the etravirine group versus $40 \%$ of those randomized to placebo (both combined with an optimized background regimen that included darunavir/ritonavir) [39]. The antiviral activity of etravirine is reduced in the presence of three or more specific resistance-associated mutations [49].

Maraviroc and raltegravir, a CCR5 receptor antagonist and an integrase inhibitor, respectively, were the first available new-class options that could be given orally. While being an attractive agent in terms of its effectiveness and tolerability, maraviroc is limited to use in the treatment of patients with CCR5-tropic virus, which is an issue in treatment-experienced patients [50]. In the pooled results of the MOTIVATE 1 and 2 studies, the 48-week rates of virologic suppression to $<50$ copies $/ \mathrm{mL}$ for treatmentexperienced subjects with R5-tropic virus were $43 \%$ with maraviroc once daily and $46 \%$ with maraviroc twice daily versus $17 \%$ with placebo (all with an optimized background regimen) [35]. Economic evaluations of the MOTIVATE 1 and 2 studies have compared maraviroc plus optimized background therapy to optimized background alone. The incremental cost-effectiveness ratios per QALY gained were $€ 23,457$ and US\$42,429 in analyses conducted in Spain and Mexico, respectively [51, 52]. The incremental costeffectiveness ratio was found to be somewhat lower (more favorable) when maraviroc was modeled in individuals whose HIV was susceptible to two or fewer components of the background regimen and higher (less favorable) in individuals with HIV susceptible to three or more regimen components $[52,53]$.

Use of the integrase inhibitor raltegravir is not restricted by tropism, and the drug is effective against virus resistant to other drug classes. Given orally twice daily, it is relatively safe and well-tolerated, with minimal toxicity and drug interactions [54]. In the combined BENCHMRK 1 and 2 study results, the 48 -week rates of virologic suppression to $<50$ copies $/ \mathrm{mL}$ for treatment-experienced subjects with drug-resistant HIV were $62 \%$ with raltegravir versus $33 \%$ with placebo (both combined with an optimized background regimen) [36]. A pair of studies from Spain and Switzerland used data from the BENCHMRK 1 and 2 trials to assess the long-term cost-effectiveness of raltegravir plus background therapy as compared to background therapy alone. Incremental cost-effectiveness ratios for three years of treatment were calculated to be $€ 22,908$ and 42,751 Swiss francs in the two studies, respectively, and increased with longer treatment durations $[55,56]$. By 2009, many patients receiving enfuvirtide in rescue therapy regimens were switched to raltegravir [57-60], with a significant improvement in patient acceptability and cost. Given the inconvenience of twice daily injections and the availability of raltegravir and other oral agents effective against MDR virus, the clinical role of enfuvirtide diminished, and it is seldom used today.

When they became approved and available, these newer agents were somewhat more expensive than the previous antiretrovirals (except enfuvirtide). The DUET, MOTIVATE, and BENCHMRK trials were conducted in treatmentexperienced patients, where complex and expensive drug combinations are typically required. The average annual per 
patient cost of antiretrovirals for the active plus optimized background regimen arm versus placebo plus optimized background regimen was US $\$ 47,324$ versus 38,267 in the DUET Trials, US $\$ 46,633$ versus 36,404 in MOTIVATE, and US\$ 45,484 versus 34,585 in BENCHMRK. Of note, in the three trials, the highest treatment costs were from nucleoside analogues (29-30\% of total costs) and enfuvirtide (22-25\% of total costs) [61]. The ability to design an effective regimen for a patient with MDR virus using fewer drugs than the previous multiple drug rescue therapy regimens permitted more cost-effective therapy. In addition, the improved safety and tolerability of most of these newer agents resulted in lower overall health care costs for the treatment of these individuals. By 2008 it became possible to successfully treat patients with MDR virus with a regimen including three active new agents: ritonavir-boosted darunavir, etravirine, and raltegravir, with or without partially effective nucleosides $[62,63]$.

\section{Future: 2012 and Beyond}

Despite evidence of ongoing risk behavior in patients infected with drug-resistant HIV [64], the spectre of widespread transmission of multidrug-resistant HIV has not materialized. This is probably related at least in part to the reduced fitness of multiply-mutant strains [65]. As combination antiretroviral regimens have become more potent in suppressing viral replication and genotypic resistance testing prior to treatment has become standard of care, the majority of HIV drug resistance emerges in the setting of incomplete adherence. First-line HAART regimens are becoming more convenient, more forgiving to missed doses, and better tolerated, so the emergence of resistance continues to become less frequent. As well, the long-term durability of modern HAART regimens is increasing [66].

On the other hand, the significant flourishing of new antiretroviral drugs and new drug classes that has occurred over the past several years is unlikely to be duplicated in the future. Fewer new agents are being developed for treating HIV, and some of them (e.g., rilpivirine) are aimed specifically at first-line therapy of treatment-naïve patients [67]. Two new integrase inhibitors, elvitegravir and dolutegravir, may play a role in treatment of drug-resistant HIV $[68,69]$; however, elvitegravir demonstrates significant cross-resistance with raltegravir and is therefore unlikely to be effective for patients who have failed a raltegravirbased regimen [70]. Given the paucity of new antiretroviral drugs in the pipeline, the agents that are currently available will need to continue to control HIV replication for many years. Furthermore, many regimens in current use include drugs with a low genetic barrier to resistance (e.g., efavirenz, raltegravir), meaning that resistant HIV mutants can emerge relatively quickly as a consequence of virologic failure $[49,71,72]$. Therefore, strategic use of the available drugs and cautious management of patients will be critical for successful HIV management in the future. Adherence assessment and counseling will need to be integrated into routine clinical visits prior to and throughout antiretroviral treatment, in order to avert the emergence of drug-resistant HIV. In addition, routine pretreatment genotypic testing to detect primary resistance, regular viral load monitoring, and early genotypic testing in cases of virologic failure will be particularly important to prevent accumulation of resistance-associated mutations.

The burden of resistance could increase if new agents are not made available or their introduction is staggered, resulting in suboptimal regimens or functional monotherapy. This issue is particularly relevant to developing countries, where options for second-line and salvage therapy are limited by scarcity of resources; the newer drugs that are effective against drug-resistant HIV are costly and often not available in generic formulations. Furthermore, in these resource-limited settings, access to viral load monitoring and resistance testing may be restricted, if available at all. The absence of these laboratory tools to monitor the ongoing effectiveness of antiretroviral therapy can lead to significant delays in the diagnosis and management of virologic failure, with devastating consequences $[64,73]$. The inevitable emergence of HIV drug resistance in these settings is a particular concern at a time when it has been recognized that sustained full suppression of viral replication is crucial, both to optimize the individual benefit of HAART and to decrease HIV transmission [74-77]. The optimal rollout of HAART in both rich and resource-limited settings will require careful planning to ensure access to the best available antiretroviral regimens and to the appropriate technologies to monitor their use.

\section{References}

[1] R. S. Hogg, D. R. Bangsberg, V. D. Lima et al., "Emergence of drug resistance is associated with an increased risk of death among patients first starting HAART," PLoS Medicine, vol. 3, no. 9, pp. 1570-1578, 2006.

[2] D. D. Richman, S. C. Morton, T. Wrin et al., "The prevalence of antiretroviral drug resistance in the United States," AIDS, vol. 18, no. 10, pp. 1393-1401, 2004.

[3] V. D. Lima, P. R. Harrigan, M. Sénécal et al., "Epidemiology of antiretroviral multiclass resistance," American Journal of Epidemiology, vol. 172, no. 4, pp. 460-468, 2010.

[4] R. M. Gulick, J. W. Mellors, D. Havlir et al., "Treatment with indinavir, zidovudine, and lamivudine in adults with human immunodeficiency virus infection and prior antiretroviral therapy," The New England Journal of Medicine, vol. 337, no. 11, pp. 734-739, 1997.

[5] J. S. G. Montaner, P. Reiss, D. Cooper et al., "A randomized double-blind trial comparing combinations of nevirapine, didanosine, and zidovudine for HIV-infected patients: the INCAS trial," JAMA, vol. 279, no. 12, pp. 930-937, 1998.

[6] D. W. Cameron, A. J. Japour, Y. Xu et al., "Ritonavir and saquinavir combination therapy for the treatment of HIV infection," AIDS, vol. 13, no. 2, pp. 213-224, 1999.

[7] C. S. Hall, C. P. Raines, S. H. Barnett, R. D. Moore, and J. E. Gallant, "Efficacy of salvage therapy containing ritonavir and saquinavir after failure of single protease inhibitor-containing regimens," AIDS, vol. 13, no. 10, pp. 1207-1212, 1999.

[8] F. Lallemand, N. Adda, V. Schneider, C. Jacomet, Y. Salhi, and W. Rozenbaum, "Prospective follow-up of 67 indinavirexperienced human immunodeficiency virus-infected and 
AIDS patients treated with the ritonavir/saquinavir combination," Clinical Infectious Diseases, vol. 28, no. 5, pp. 1184-1185, 1999.

[9] V. Miller, C. Sabin, K. Hertogs et al., "Virological and immunological effects of treatment interruptions in HIV-1 infected patients with treatment failure," AIDS, vol. 14, no. 18, pp. 2857-2867, 2000.

[10] J. Lawrence, K. Huppler Hullsiek, L. M. Thackeray et al., "Disadvantages of structured treatment interruption persist in patients with multidrug-resistant HIV-1: final results of the CPCRA 064 study," Journal of Acquired Immune Deficiency Syndromes, vol. 43, no. 2, pp. 169-178, 2006.

[11] V. Miller, A. Cozzi-Lepri, K. Hertogs et al., "HIV drug susceptibility and treatment response to mega-HAART regimens in patients from the Frankfurt HIV cohort," Antiviral Therapy, vol. 5, no. 1, pp. 49-55, 2000.

[12] J. S. G. Montaner, P. R. Harrigan, N. Jahnke et al., "Multiple drug rescue therapy for HIV-infected individuals with prior virologic failure to multiple regimens," AIDS, vol. 15, no. 1, pp. 61-69, 2001.

[13] C. Katlama, S. Dominguez, K. Gourlain et al., "Benefit of treatment interruption in HIV-infected patients with multiple therapeutic failures: a randomized controlled trial (ANRS 097)," AIDS, vol. 18, no. 2, pp. 217-226, 2004.

[14] J. P. Lalezari, K. Henry, M. O’Hearn et al., "Enfuvirtide, an HIV-1 fusion inhibitor, for drug-resistant HIV infection in North and South America," The New England Journal of Medicine, vol. 348, no. 22, pp. 2175-2185, 2003.

[15] A. Lazzarin, B. Clotet, D. Cooper et al., "Efficacy of enfuvirtide in patients infected with drug-resistant HIV-1 in Europe and Australia," The New England Journal of Medicine, vol. 348, no. 22, pp. 2186-2195, 2003.

[16] M. Nelson, K. Arastéh, B. Clotet et al., "Durable efficacy of enfuvirtide over 48 weeks in heavily treatment-experienced HIV-1-infected patients in the T-20 versus optimized background regimen only 1 and 2 clinical trials," Journal of Acquired Immune Deficiency Syndromes, vol. 40, no. 4, pp. 404412, 2005.

[17] M. Harris, P. R. Harrigan, and J. S. G. Montaner, "Chapter 46: Antiretroviral Therapy of Drug-resistant HIV," in Global HIV/AIDS Medicine, P. A. Volberding, M. A. Sande, J. Lange, W. C. Greene, and J. E. Gallant, Eds., Saunders Elsevier, 2007.

[18] R. Steinbrook, "HIV infection-a new drug and new costs," The New England Journal of Medicine, vol. 348, no. 22, pp. 2171-2172, 2003.

[19] K. T. Tashima and C. C. J. Carpenter, "Fusion inhibition-a major but costly step forward in the treatment of HIV-1," The New England Journal of Medicine, vol. 348, no. 22, pp. 22492250, 2003.

[20] Bureau of Labor and Statistics. Consumer price index-medical care component, http://www.bls.gov/cpi/.

[21] Drug Topics Red Book, Medical Economics, Montvale, NJ, USA, 2001.

[22] P. A. Pham, "Drug profile: fosamprenavir (Lexiva)," The Hopkins HIV Report, vol. 15, no. 6, pp. 10-11, 2003.

[23] J. Montaner, D. Guimaraes, J. Chung, Z. Gafoor, M. Salgo, and R. DeMasi, "Prognostic staging of extensively pretreated patients with advanced HIV-1 disease," HIV Clinical Trials, vol. 6, no. 6, pp. 281-290, 2005.

[24] Drug Topics Red Book, Medical Economics, Montvale, NJ, USA, 2004.

[25] P. E. Sax, E. Losina, M. C. Weinstein et al., "Cost-effectiveness of enfuvirtide in treatment-experienced patients with advanced HIV disease," Journal of Acquired Immune Deficiency Syndromes, vol. 39, no. 1, pp. 69-77, 2005.

[26] J. Hornberger, J. M. Kilby, N. Wintfeld, and J. Green, "Costeffectiveness of enfuvirtide in HIV therapy for treatmentexperienced patients in the United States," AIDS Research and Human Retroviruses, vol. 22, no. 3, pp. 240-247, 2006.

[27] World Health Organization. Choosing interventions that are cost-effective (WHOCHOICE): cost-effectiveness thresholds, http://www.who.int/choice/costs/CER_thresholds/en/index .html.

[28] International Monetary Fund, World Economic Outlook Database, International Monetary Fund, Washington, DC, USA, 2008, http://www.imf.org/external/pubs/ft/weo/2008/ 01/weodata/weorept.aspx?sy=2008\&ey=2008\&scsm=1\&ssd= $1 \&$ sort $=$ country.

[29] B. Trottier, S. Walmsley, J. Reynes et al., "Safety of enfuvirtide in combination with an optimized background of antiretrovirals in treatment-experienced HIV-1-infected adults over 48 weeks," Journal of Acquired Immune Deficiency Syndromes, vol. 40, no. 4, pp. 413-421, 2005.

[30] M. Harris, R. Joy, G. Larsen et al., "Enfuvirtide plasma levels and injection site reactions using a needle-free gas-powered injection system (Biojector)," AIDS, vol. 20, no. 5, pp. 719723,2006

[31] C. B. Hicks, P. Cahn, D. A. Cooper et al., "Durable efficacy of tipranavir-ritonavir in combination with an optimised background regimen of antiretroviral drugs for treatmentexperienced HIV-1-infected patients at 48 weeks in the Randomized Evaluation of Strategic Intervention in multidrug reSistant patients with Tipranavir (RESIST) studies: an analysis of combined data from two randomised open-label trials," The Lancet, vol. 368, no. 9534, pp. 466-475, 2006.

[32] B. Clotet, N. Bellos, J. M. Molina et al., "Efficacy and safety of darunavir-ritonavir at week 48 in treatment-experienced patients with HIV-1 infection in POWER 1 and 2: a pooled subgroup analysis of data from two randomised trials," The Lancet, vol. 369, no. 9568, pp. 1169-1178, 2007.

[33] J. V. Madruga, P. Cahn, B. Grinsztejn et al., "Efficacy and safety of TMC125 (etravirine) in treatment-experienced HIV1-infected patients in DUET-1: 24-week results from a randomised, double-blind, placebo-controlled trial," The Lancet, vol. 370, no. 9581, pp. 29-38, 2007.

[34] A. Lazzarin, T. Campbell, B. Clotet et al., "Efficacy and safety of TMC125 (etravirine) in treatment-experienced HIV1-infected patients in DUET-2: 24-week results from a randomised, double-blind, placebo-controlled trial," The Lancet, vol. 370, no. 9581, pp. 39-48, 2007.

[35] R. M. Gulick, J. Lalezari, J. Goodrich et al., "Maraviroc for previously treated patients with R5 HIV-1 infection," The New England Journal of Medicine, vol. 359, no. 14, pp. 1429-1441, 2008.

[36] R. T. Steigbigel, D. A. Cooper, P. N. Kumar et al., "Raltegravir with optimized background therapy for resistant HIV-1 infection," The New England Journal of Medicine, vol. 359, no. 4, pp. 339-354, 2008.

[37] C. Katlama, R. Esposito, J. M. Gatell et al., "Efficacy and safety of TMC114/ritonavir in treatment-experienced HIV patients: 24-Week results of POWER 1," AIDS, vol. 21, no. 4, pp. 395402, 2007.

[38] R. Haubrich, D. Berger, P. Chiliade et al., "Week 24 efficacy and safety of TMC114/ritonavir in treatment-experienced HIV patients," AIDS, vol. 21, no. 6, pp. F11-F18, 2007.

[39] C. Katlama, R. Haubrich, J. Lalezari et al., "Efficacy and safety of etravirine in treatment-experienced, HIV-1 patients: pooled 
48 week analysis of two randomized, controlled trials," AIDS, vol. 23, no. 17, pp. 2289-2300, 2009.

[40] G. A. A. Hubben, J. M. Bos, C. A. Veltman-Starkenburg et al., "Cost-effectiveness of tipranavir versus comparator protease inhibitor regimens in HIV infected patients previously exposed to antiretroviral therapy in the Netherlands," Cost Effectiveness and Resource Allocation, vol. 5, article 15, 2007.

[41] K. N. Simpson, G. Roberts, C. B. Hicks, and H. W. Finnern, "Cost-effectiveness of tipranavir in treatment-experienced HIV patients in the United States," HIV Clinical Trials, vol. 9, no. 4, pp. 225-237, 2008.

[42] K. M. Chan-Tack, K. A. Struble, and D. B. Birnkrant, "Intracranial hemorrhage and liver-associated deaths associated with tipranavir/ritonavir: review of cases from the FDA's adverse event reporting system," AIDS Patient Care and STDs, vol. 22, no. 11, pp. 843-850, 2008.

[43] J. V. Madruga, D. Berger, M. McMurchie et al., "Efficacy and safety of darunavir-ritonavir compared with that of lopinavirritonavir at 48 weeks in treatment-experienced, HIV-infected patients in TITAN: a randomised controlled phase III trial," The Lancet, vol. 370, no. 9581, pp. 49-58, 2007.

[44] J. Mauskopf, L. Annemans, A. M. Hill, and E. Smets, "A review of economic evaluations of darunavir boosted by low-dose ritonavir in treatment-experienced persons living with HIV infection," PharmacoEconomics, vol. 28, no. 1, pp. 1-16, 2010.

[45] E. Ribera and A. Curran, "Double-boosted protease inhibitor antiretroviral regimens: what role?" Drugs, vol. 68, no. 16, pp. 2257-2267, 2008.

[46] S. M. Hammer, F. Vaida, K. K. Bennett et al., "Dual vs single protease inhibitor therapy following antiretroviral treatment failure: a randomized trial," JAMA, vol. 288, no. 2, pp. 169180, 2002.

[47] C. Cohen, R. Liporace, E. de Jesus et al., "Use of twicedaily darunavir as a substitution for dual-boosted PIs in virologically suppressed patients: primary endpoint results of a pilot randomized clinical trial," in Proceedings of the 12th European AIDS Conference, Cologne, Germany, November 2009, Abstract PS4/2.

[48] M. Harris, M. Hull, P. Ochoa, A. Toulson, and J. Montaner, "Immediate vs. deferred substitution of dual boosted protease inhibitors (PIs) with darunavir/ritonavir (DRV/r)," Canadian Journal of Infectious Diseases \& Medical Microbiology, vol. 20, supplement B, p. 16B, Abstract O013, 2009.

[49] V. A. Johnson, V. Calvez, H. F. Günthard et al., "2011 update of the drug resistance mutations in HIV-1," Topics in Antiviral Medicine, vol. 19, no. 4, pp. 156-164, 2011.

[50] T. J. Wilkin, Z. Su, D. R. Kuritzkes et al., "HIV type 1 chemokine coreceptor use among antiretroviral-experienced patients screened for a clinical trial of a CCR5 inhibitor: AIDS Clinical Trial Group A5211," Clinical Infectious Diseases, vol. 44, no. 4, pp. 591-595, 2007.

[51] S. Moreno, J. González, I. Lekander et al., "Cost-effectiveness of optimized background therapy plus maraviroc for previously treated patients with R5 HIV-1 infection from the perspective of the Spanish health care system," Clinical Therapeutics, vol. 32, no. 13, pp. 2232-2245, 2010.

[52] I. Contreras-Hernandez, D. Becker, J. Chancellor et al., "Cost-effectiveness of maraviroc for antiretroviral treatmentexperienced HIV-infected individuals in Mexico," Value in Health, vol. 13, no. 8, pp. 903-914, 2010.

[53] F. Kühne, J. Chancellor, P. Mollon, D. Myers, M. Louie, and W. Powderly, "A microsimulation of the cost-effectiveness of maraviroc for antiretroviral treatment-experienced HIVinfected individuals," HIV Clinical Trials, vol. 11, no. 2, pp. 80-99, 2010.

[54] M. Harris, "Raltegravir: its use in the treatment of HIV infection," Clinical Medicine: Therapeutics, vol. 1, pp. 183-197, 2009.

[55] M. A. Chaudhary, S. Moreno, R. N. Kumar, G. Nocea, and E. Elbasha, "Cost-effectiveness analysis of raltegravir in treatment-experienced HIV type 1-infected patients in Spain," AIDS Research and Human Retroviruses, vol. 25, no. 7, pp. 679$689,2009$.

[56] E. E. Elbasha, T. Szucs, M. A. Chaudhary et al., "Costeffectiveness of raltegravir in antiretroviral treatmentexperienced HIV-1-infected patients in Switzerland," HIV Clinical Trials, vol. 10, no. 4, pp. 233-253, 2009.

[57] M. Harris, G. Larsen, and J. S. G. Montaner, "Outcomes of multidrug-resistant patients switched from enfuvirtide to raltegravir within a virologically suppressive regimen," AIDS, vol. 22, no. 10, pp. 1224-1226, 2008.

[58] N. De Castro, J. Braun, I. Charreau et al., "Switch from enfuvirtide to raltegravir in virologically suppressed multidrugresistant HIV-1-infected patients: a randomized open-label trial," Clinical Infectious Diseases, vol. 49, no. 8, pp. 1259-1267, 2009.

[59] W. Towner, D. Klein, H. L. Kerrigan, S. Follansbee, K. Yu, and M. Horberg, "Virologic outcomes of changing enfuvirtide to raltegravir in HIV-1 patients well controlled on an enfuvirtide based regimen: 24-Week results of the CHEER study," Journal of Acquired Immune Deficiency Syndromes, vol. 51, no. 4, pp. 367-373, 2009.

[60] J. R. Santos, J. M. Llibre, E. Ferrer et al., "Efficacy and safety of switching from enfuvirtide to raltegravir in patients with virological suppression," HIV Clinical Trials, vol. 10, no. 6, pp. 432-438, 2009.

[61] A. M. Hill, M. Cho, and J. M. Mrus, "The costs of full suppression of plasma HIV RNA in highly antiretroviralexperienced patients," AIDS Reviews, vol. 13, no. 1, pp. 41-48, 2011.

[62] Y. Yazdanpanah, C. Fagard, D. Descamps et al., "High rate of virologic suppression with raltegravir plus etravirine and darunavir/ ritonavir among treatment-experienced patients infected with multidrug-resistant HIV: results of the ANRS 139 TRIO trial," Clinical Infectious Diseases, vol. 49, no. 9, pp. 1441-1449, 2009.

[63] A. Imaz, S. Villar del Saz, M. A. Ribas et al., "Raltegravir, etravirine, and ritonavir-boosted darunavir: a safe and successful rescue regimen for multidrug-resistant HIV-1 infection," Journal of Acquired Immune Deficiency Syndromes, vol. 52, no. 3, pp. 382-386, 2009.

[64] M. J. Kozal, "Drug-resistant human immunodefiency virus," Clinical Microbiology and Infection, vol. 15, supplement s1, pp. 69-73, 2009.

[65] B. G. Brenner, J. P. Routy, M. Petrella et al., "Persistence and fitness of multidrug-resistant human immunodeficiency virus type 1 acquired in primary infection," Journal of Virology, vol. 76, no. 4, pp. 1753-1761, 2002.

[66] V. S. Gill, V. D. Lima, W. Zhang et al., "Improved virological outcomes in British Columbia concomitant with decreasing incidence of HIV type 1 drug resistance detection," Clinical Infectious Diseases, vol. 50, no. 1, pp. 98-105, 2010.

[67] Edurant (rilpivirine) Tablets; Full Prescribing Information. Tibotec, Inc. 2011.

[68] A. R. Zolopa, D. S. Berger, H. Lampiris et al., "Activity of elvitegravir, a once-daily integrase inhibitor, against resistant 
HIV type 1: results of a phase 2, randomized, controlled, doseranging clinical trial," Journal of Infectious Diseases, vol. 201, no. 6, pp. 814-822, 2010.

[69] J. Eron, P. Kumar, A. Lazzarin et al., "DTG in subjects with HIV exhibiting RAL resistance: functional monotherapy results of VIKING study cohort II," in Proceedings of the 18th Conference on Retroviruses and Opportunistic Infections (CROI '11), Boston, Mass, USA, February 2011, Abstract 151LB.

[70] C. Garrido, J. Villacian, N. Zahonero et al., "Broad phenotypic cross-resistance to elvitegravir in HIV-infected patients failing on raltegravir-containing regimens," Antimicrobial Agents and Chemotherapy, vol. 56, no. 6, pp. 2873-2878, 2012.

[71] J. J. Eron, B. Young, D. A. Cooper et al., "Switch to a raltegravir-based regimen versus continuation of a lopinavirritonavir-based regimen in stable HIV-infected patients with suppressed viraemia (SWITCHMRK 1 and 2): two multicentre, double-blind, randomised controlled trials," The Lancet, vol. 375, no. 9712, pp. 396-407, 2010.

[72] F. Baldanti, S. Paolucci, R. Gulminetti, M. Brandolini, G. Barbarini, and R. Maserati, "Early emergence of raltegravir resistance mutations in patients receiving HAART salvage regimens," Journal of Medical Virology, vol. 82, no. 1, pp. 116$122,2010$.

[73] C. Hawkins and R. L. Murphy, "Management of antiretroviral failure and resistance in developing countries," Current Opinion in HIV and AIDS, vol. 4, no. 6, pp. 538-544, 2009.

[74] J. S. Montaner, R. Hogg, E. Wood et al., "The case for expanding access to highly active antiretroviral therapy to curb the growth of the HIV epidemic," The Lancet, vol. 368, no. 9534, pp. 531-536, 2006.

[75] J. S. Montaner, V. D. Lima, R. Barrios et al., "Association of highly active antiretroviral therapy coverage, population viral load, and yearly new HIV diagnoses in British Columbia, Canada: a population-based study," The Lancet, vol. 376, no. 9740, pp. 532-539, 2010.

[76] J. S. Montaner, "Treatment as prevention-a double hattrick," The Lancet, vol. 378, no. 9787, pp. 208-209, 2011.

[77] M. S. Cohen, Y. Q. Chen, M. McCauley et al., "Prevention of HIV-1 infection with early antiretroviral therapy," The New England Journal of Medicine, vol. 365, no. 6, pp. 493-505, 2011. 


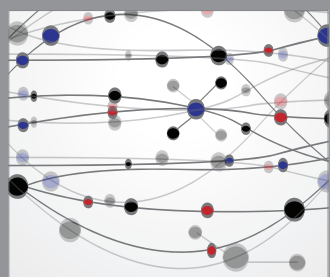

The Scientific World Journal
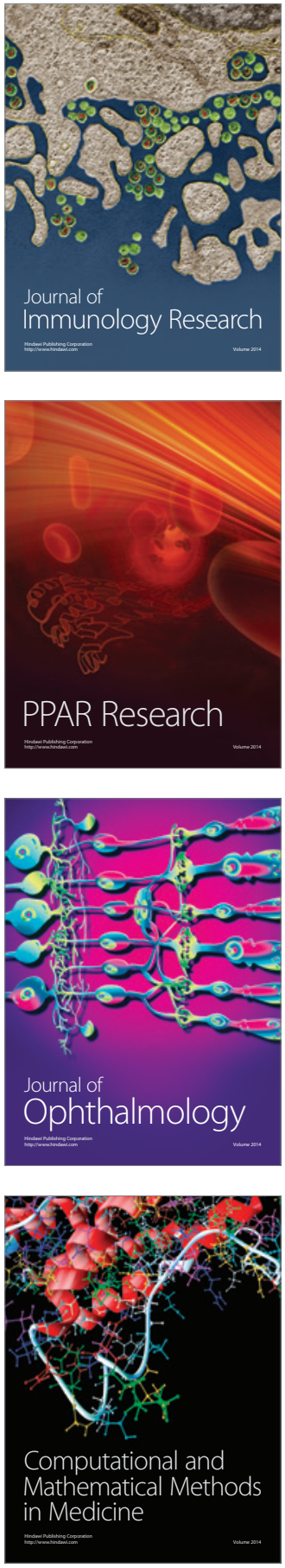

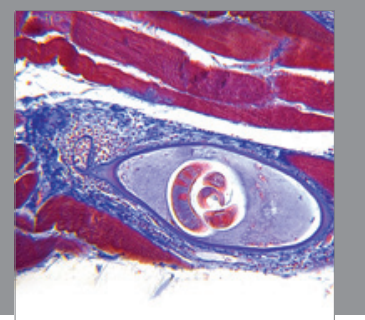

Gastroenterology

Research and Practice
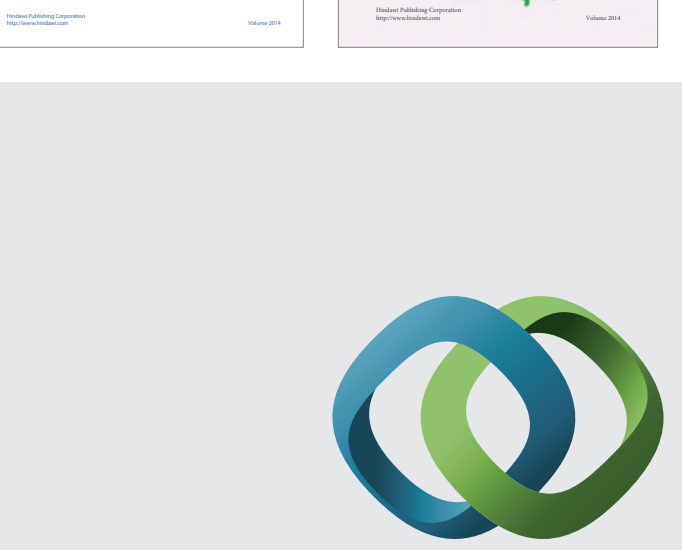

\section{Hindawi}

Submit your manuscripts at

http://www.hindawi.com
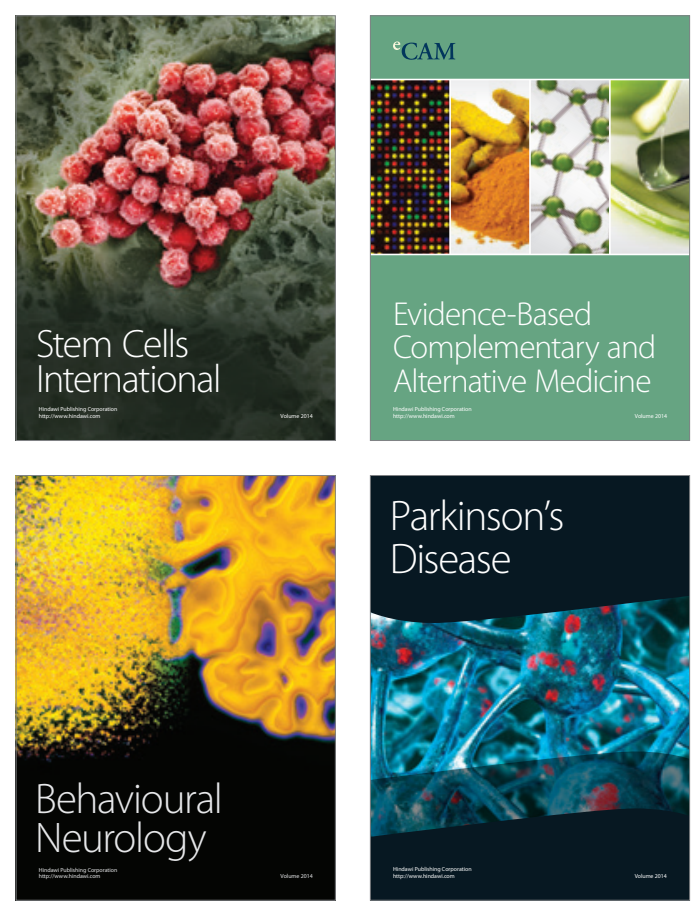

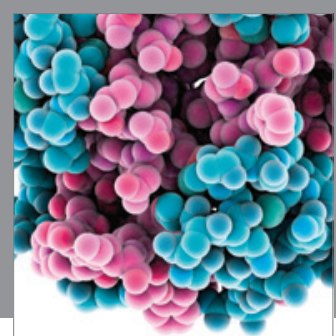

Journal of
Diabetes Research

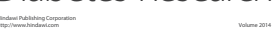

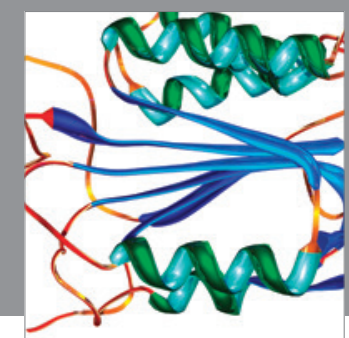

Disease Markers
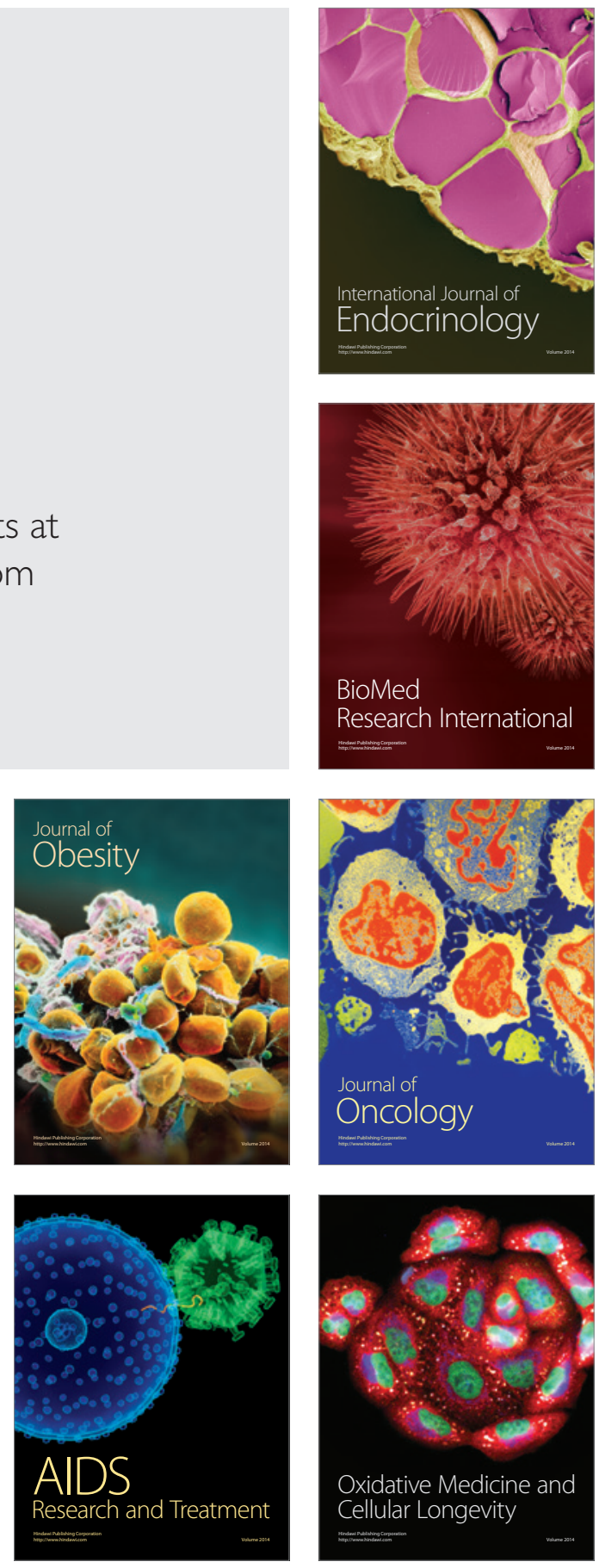